\title{
Development of a Selective Medium for the Fungal Pathogen Cylindrocarpon destructans Using Radicicol
}

\author{
Yunhee Kang ${ }^{1}$, Seung-Ho Lee ${ }^{2}$ and Jungkwan Lee ${ }^{1 *}$ \\ ${ }^{1}$ Department of Applied Biology, Dong-A University, Busan 604-714, Korea \\ ${ }^{2}$ Ginseng Research Division, National Institute of Horticultural and Herbal Science, Eumseong 369-873, Korea
}

(Received on August 5, 2014; Revised on September 3, 2014; Accepted on September 4, 2014)

The soil-borne ascomycete fungus Cylindrocarpon destructans causes ginseng root rot disease and produces various secondary metabolites such as brefeldin $A$ and radicicol. The slow growth of this fungus compared with other plant pathogenic and saprophytic fungi in soil disturbs isolation of this fungus from soil and infected ginseng. In this study, we developed a selective medium for $C$. destructans using radicicol produced by this fungus. Supplementing $50 \mathrm{mg} / \mathrm{L}$ of radicicol to medium inhibited the mycelia growth of other fungi including Botrytis cinerea, Rhizoctonia solani and Alternaria panax, but did not affect the growth of $C$. destructans. In addition, conidia germination of other fungal species except for $C$. destructans was inhibited in submerged culture supplemented with radicicol. This medium provides a very efficient tool for isolating $C$. destructans and also can be used as an enrichment medium for this fungus.

Keywords : Cylindrocarpon destructans, Fusarium solani, ginseng root rot, radicicol, selective medium

Ginseng (Panax ginseng Meyer) has been widely used for traditional medicine in East Asia including Korea, China and Japan. Stable ginseng production has been often threatened by root rot which is associated with fungal pathogens such as Cylindrocarpon destructans, Fusarium solani, Rhizoctonia solani, and Pythium ultimum (Chung, 1975; Punja, 1997; Shin et al., 2012). Of these fungal pathogens, the homothallic ascomycete fungus $C$. destructans (teleomorph Nectria radicicola) is the main causal agent for root rot disease in ginseng cultivation area worldwide (Ahn and Lee, 2001; Chung, 1975; Reeleder and Brammall, 1994). This

*Corresponding author.

Phone) +82-51-200-7554, FAX) +82-51-200-7505

E-mail) jungle@dau.ac.kr fungus is also known as one of the most detrimental factors in replant failure in ginseng cultivation and replant failure might be caused by the increase of pathogenic population of the fungus among the soil microbial community in field (Chung, 1975; Hopkins et al., 1992).

Isolation of fungal pathogens from infected plants is important for various research areas including population genetics, disease diagnosis, and disease forecasting. The slow vegetative growth of $C$. destructans compared with other soil-borne fungi such as $F$. solani and $R$. solani which are widely distributed in soil and are often associated with ginseng root rot disturbs the isolation of this fungus from infected ginseng and soil. The selective medium for $C$. destructans was developed as supplementing fungicides such as metalaxyl and pentachloronitrobenzene (PCNB) to czapek dox agar (CDA) adjusted with pH 10 (Sweetingham, 1983). This medium efficiently suppresses contamination of fungi belonging to either oomycetes or zygomycetes, but it is not efficient to inhibit the growth of fungi belonging to ascomycetes and deuteromycetes.

Previously, we developed a selective medium for Fusarium species using toxoflavin produced by the bacterial plant pathogen Burkholderia glumae (Jung et al., 2013), suggesting that natural compounds can be used as an ingredient for a selective medium to isolate specific fungal species. For the development of a selective medium for $C$. destructans, we had focused on the secondary metabolites, brefeldin $\mathrm{A}$ and radicicol, produced by this fungus. Brefeldin $\mathrm{A}$ is a lactone antibiotic and inhibits protein transport from the endoplasmic reticulum to the Golgi apparatus (Klausner and Donaldson, 1992). Radicicol binds to heat shock protein 90 and alters its functions, resulting in antibiotic effect (Schulte et al., 1998).

We hypothesized that $C$. destructans strains are resistant to either brefeldin A or radicicol because this fungus can produce both secondary metabolites, but other fungal species not producing these compounds are sensitive to these toxins. Based on this hypothesis, we showed that the 
medium supplemented with radicicol efficiently inhibited other plant pathogenic and saprophytic fungal species but C. destructans strains were well grown, suggesting that this medium can be widely used by fungal isolation and disease diagnosis.

For this study, soil-borne fungal pathogens, C. destructans, $F$. solani, and $R$. solani strains were isolated from ginseng roots collected at Eumseong in 2013. Each strain belonging to $C$. destructans and $F$. solani was purified by single spore isolation and $R$. solani strains were subcultured from the growing margins. The strains were identified based on morphological characteristics including vegetative growth and conidia production, and phylogenic analysis. Fungal genomic DNA was extracted from mycelia cultured on complete medium (CM) as previously described (Chi et al., 2009; Leslie and Summerell, 2006). The internal transcribed spacer of each strain was amplified through polymerase chain reaction with a pair of primer as previously described (White et al., 1990), and the amplicon was directly sequenced at the National Instrumentation Center for Environmental Management (Seoul, Korea) after purification using QIAquick PCR purification kit (Qiagen). The sequences were compared to the NCBI database with standard nucleotide BLAST (http://blast.ncbi.nlm.nih. gov/Blast.cgi /). Other fungal strains, C. destructans (KACC No.41077), Alternaria panax (KACC No.42461), Sclerotinia sclerotiorum (KACC No.42265), and P. ultimum (KACC No.42265) were provided from RDA-Genebank Information Center (Suwon, Korea), and Botrytis cinerea was provided from Center for Fungal Genetics Resources (Seoul, Korea).

To test the rate of mycelial growth of different fungal strains compared to that of $C$. destructans, a mycelial agar plug (5 mm diameter) was inoculated on potato dextrose agar (PDA), minimal medium (MM) and CM (Leslie and Summerell, 2006) and the growth rate was measured every $24 \mathrm{~h}$. The experiment was repeated three times with three replicates, and Tukey test using SPSS 12.0 software (SPSS Inc., Chicago, USA) was performed to examine the significant differences $(P<0.05)$ among the mean values of samples. The mycelial growth of $C$. destructans was slow compared to other fungi including $R$. solani, F. solani, B. cinerea, A. panax, S. sclerotiorum, and P. ultimum. To maximize the growth of $C$. destructans compared to other fungal species, we first check the optimized growth condition at various temperatures. At $20^{\circ} \mathrm{C}$, the mycelial growth of $C$. destructans was highest but the growth of other fungal species surpassed that of $C$. destructans at this temperature (Fig. 1). At $15^{\circ} \mathrm{C}$, the mycelial growth of fungi except for B. cinerea, S. sclerotiorum, and P. ultimum was

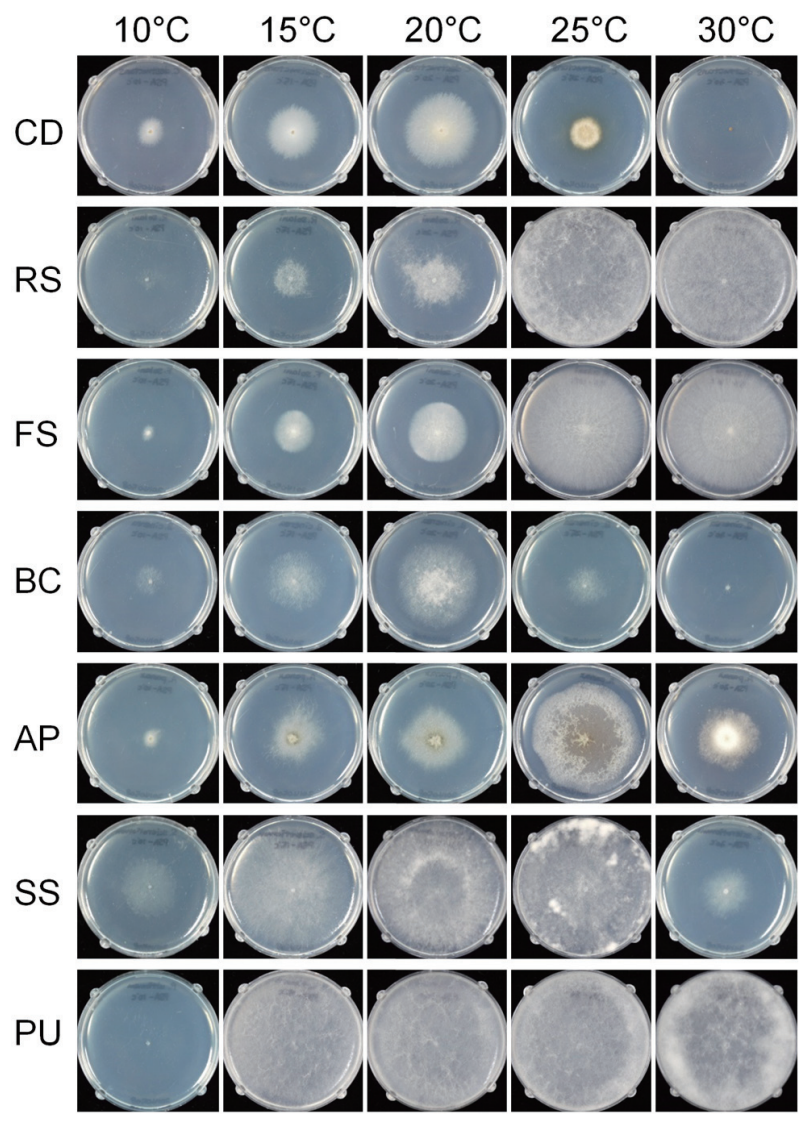

Fig. 1. Growth optimization of Cylindrocarpon destructans compared to various fungal species isolated from soil. Each fungal strain was inoculated on potato dextrose agar and incubated in various temperatures. Photographs were taken $4 \mathrm{~d}$ after inoculation. CD, C. destructans; RS, Rhizoctonia solani; FS, Fusarium solani; BC, Botrytis cinerea; AP, Alternaria panax; SS, Sclerotinia sclerotiorum; PU, Pythium ultimum.

dramatically reduced, and $F$. solani growth was also slower than that incubated at $20^{\circ} \mathrm{C}$. This growth pattern based on the incubation temperatures was not significantly different among PDA, MM, and CM. Taken together, this result suggested that cultivation at $15^{\circ} \mathrm{C}$ allows better growth of $C$. destructans than at $20^{\circ} \mathrm{C}$ compared to $F$. solani.

To test whether soil-borne fungal pathogens are resistant to radicicol and brefeldin A, all strains were inoculated on MM with various concentration of radicicol or brefeldin A (Sigma Aldrich). The compounds were dissolved in methanol and added into autoclaved media after cooling down by $50^{\circ} \mathrm{C}$. After inoculating each fungal strain on to the medium, the plates were incubated at 10, 15, 20, and $25^{\circ} \mathrm{C}$ and mycelial growth was measured every $48 \mathrm{~h}$ until $8 \mathrm{~d}$ after inoculation. The mycelial growth of other fungal species except for $C$. destructans and $F$. solani was slightly inhibited at $50 \mathrm{mg} / \mathrm{l}$ brefeldin A. At $50 \mathrm{mg} / \mathrm{l}$ radicicol, the 

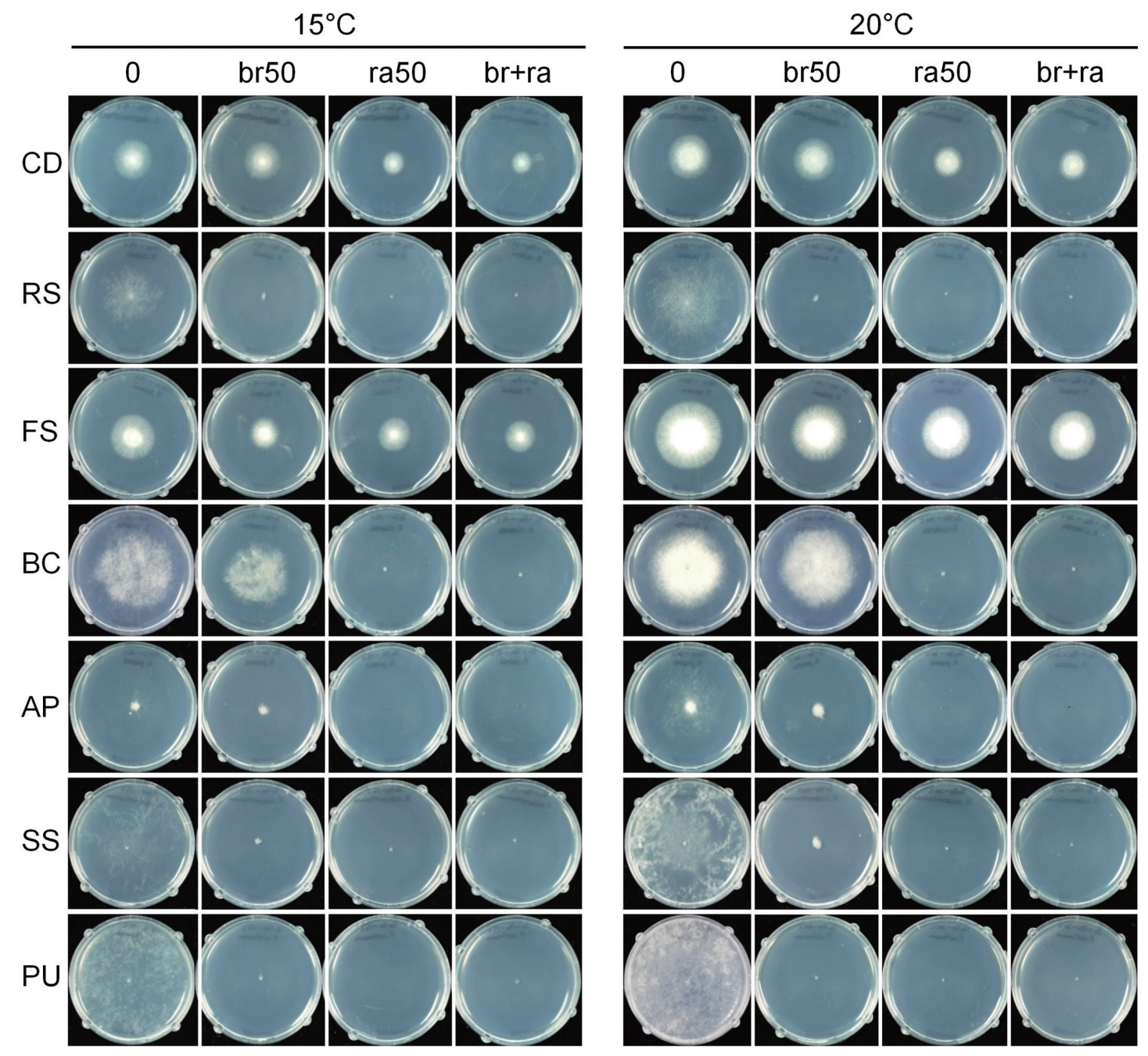

Fig. 2. Toxicity test of brefeldin $\mathrm{A}$ and radicicol against various fungal species at $15^{\circ} \mathrm{C}$ and $20^{\circ} \mathrm{C}$. Each strain was inoculated on minimal medium supplemented with either $50 \mathrm{mg} / 1$ brefeldin A or $50 \mathrm{mg} / 1$ radicicol. Photographs were taken $4 \mathrm{~d}$ after inoculation. 0, medium without brefeldin A and radicicol; br50, medium with $50 \mathrm{mg} / \mathrm{l}$ brefeldin $\mathrm{A}$; ra50, medium with $50 \mathrm{mg} / \mathrm{l}$ radicicol; br+ ra, medium with both $50 \mathrm{mg} / \mathrm{l}$ brefeldin A and $50 \mathrm{mg} / 1$ radicicol; CD, Cylindrocarpon destructans; RS, Rhizoctonia solani; FS, Fusarium solani; BC, Botrytis cinerea; AP, Alternaria panax; SS, Sclerotinia sclerotiorum; PU, Pythium ultimum.

mycelial growth of all fungal strains tested except for $C$. destructans and $F$. solani was completely inhibited in all incubation temperature tested (Fig. 2). The inhibition effect at the medium containing both compounds was not significantly different from that of the medium containing only radicicol. This result showed that radicicol is an efficient ingredient for isolation of $C$. destructans and $F$. solani as inhibiting the growth of other fungal species.

To determine whether the $C$. destructans strains present in nature are resistant and able to produce the compounds, we isolated thirty-three $C$. destructans strains from infected ginseng root. When the strains were inoculated on MM containing both $50 \mathrm{mg} / 1$ of brefeldin A and radicicol, all strains grew as well as in the MM alone (data not shown), indicating that the $C$. destructans field population was resistant to these toxins. For toxin production, each strain was inoculated on sterile-rice grains and incubated at $20^{\circ} \mathrm{C}$ for four weeks as previously described (Lee et al., 2012). Each ground culture $(20 \mathrm{~g})$ was extracted with $160 \mathrm{ml}$ methanol and $80 \mathrm{ml}$ of extract after filtrating through Whatman no. 1 filter paper was concentrated to dryness. The residue was dissolved in $1 \mathrm{ml}$ of methanol and $20 \mu \mathrm{l}$ was spotted on thin-layer chromatography (TLC) plates coated with silica gel 60 (Merck, Darmstadt, Germany). All of the strains produced radicicol that the level was able to be detected by TLC analysis but the production of brefeldin A was not detected in all the strains tested. The previous study showed that the most Cylindrocarpon species isolated from soil 


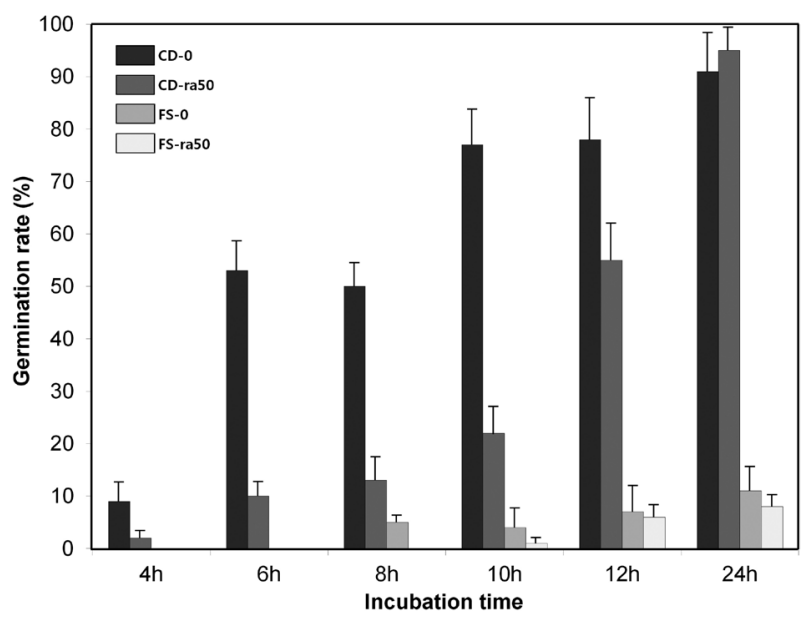

Fig. 3. Conidial germination of Cylindrocarpon destructans and Fusarium solani in minimal medium. CD-0, C. destructans without radicicol; CD-ra50, C. destructans with $50 \mathrm{mg} / \mathrm{L}$ radicicol; FS-0, F. solani without radicicol; FS-ra50, F. solani with $50 \mathrm{mg} / \mathrm{L}$ radicicol. Data are presented as mean \pm standard deviation $(n=6)$.

commonly produced brefeldin A in vitro, but its production was not detected in the diseased plant in nature (Evans, 1965). Brefeldin A has been known to have a broad spectrum of biological activity including antifungal, cytotoxic, and cancerostatic properties (Betina et al., 1966; Horakova and Betina, 1976). However, our study showed that it does not have antifungal effects on plant pathogenic fungal species isolated Korea and it is not suitable for selective isolation of $C$. destructans from soil and plants contaminated with various fungal species. On the other hand, all of the strains produced clearly detectable amount of radicicol and also the growth of various fungal species was dramatically inhibited by this toxin, suggesting that this toxin might be an important role for $C$. destructans among microbial communities in nature.

To test the effect of radicicol on conidia germination, conidia $\left(10^{5}\right.$ condia/ml $)$ of $C$. destructans and F. solani produced from potato dextrose broth and carboxylmethyl celluloase (Capellini and Peterson, 1965), respectively, were inoculated in 50-ml MM supplemented with or without $50 \mathrm{mg} / \mathrm{l}$ radicicol and conidial germination were measured every $2 \mathrm{~h}$ after incubation at either $15^{\circ} \mathrm{C}$ or $20^{\circ} \mathrm{C}$. At $20^{\circ} \mathrm{C}$, approximately $50 \%$ and $95 \%$ conidia of both species were germinated $6 \mathrm{~h}$ and $24 \mathrm{~h}$, respectively, after incubation and the germination was not affected by radicicol. At $15^{\circ} \mathrm{C}$, conidia germination of $F$. solani was dramatically reduced and supplement of radicicol accelerated the reduction. Only $10 \%$ conidia of $F$. solani were germinated even after 24 -h incubation. In the case of $C$. destructans, radicicol reduced germination rate until $12 \mathrm{~h}$ but did not affect it $24 \mathrm{~h}$ after incubation (Fig. 3). This result provided that incubation temperature and supplementation of radicicol also might be used for enrichment of $C$. destructans as delaying conidia germination of $F$. solani which is the strongest obstructer to enrich $C$. destructans.

In this study, we showed that all field strains isolated from ginseng root in Korea were able to produce radicicol and radicicol efficiently inhibited the mycelial growth of other plant pathogenic fungi including $R$. solani, $F$. solani, $B$. cinerea, A. panax, $S$. sclerotiorum, and $P$. ultimum. However, it did not inhibit the mycelial growth of $C$. destructans and $F$. solani. The mycelial growth of $F$. solani can be slow down as incubating the fungal species at $15^{\circ} \mathrm{C}$. In addition, conidial germination of $F$. solani delayed in the medium supplemented with radicicol. These results indicated that supplement of radicicol into medium provides a very efficient tool for isolating $C$. destructans and can be adopted by research groups working on genetics, biological control, and disease forecasting.

\section{Acknowledgments}

This work was supported by Rural Development Administration (PJ0101192014).

\section{References}

Ahn, I.-P. and Lee, Y.-H. 2001. A viral double-stranded RNA up regulates the fungal virulence of Nectria radicicola. Mol. Plant-Microbe Interact. 14:496-507.

Betina, V., Betinova, M. and Kutkova, M. 1966. Effects of cyanein on the growth and morphology of pathogenic fungi. Arch Mikrobiol. 55:1-16.

Cappellini, R. A. and Peterson, J. L. 1965. Macroconidium formation in submerged cultures by a nonsporulating strain of Gibberella zeae. Mycologia 57:962-966.

Chi, M.-H., Park, S.-Y., Kim, S. and Lee, Y.-H. 2009. A quick and safe method for fungal DNA extraction. Plant Pathol. J. 25:108-111.

Chung, H.-S. 1975. Studies on Cylindrocarpon destructans (Zins.) Scholten causing root rot of ginseng. Rep. Tottori Mycol. Inst. 12:127-138.

Evans, G. 1965. The antibiotic activity of Cylindrocarpon radicicola. Ph.D. thesis. University of Sydney, Sydney, Australia.

Hopkins, D. L., Lobinske, R. J. and Larkin, R. P. 1992. Selection for Fusarium oxysporum f.sp. niveum race 2 in monocultures of watermelon cultivars resistant to Fusarium wilt. Phytopathology 82:290-293.

Horakova, K. and Betina, V. 1976. Cytotoxic activity of macrocyclic metabolites from fungi. Neoplasma 24:21-27.

Jung, B., Lee, S., Ha, J., Park, J.-C., Han, S.-S., Hwang, I., Lee, 
Y.-W. and Lee, J. 2013. Development of a selective medium for the fungal pathogen Fusarium graminearum using toxoflavin produced by the bacterial pathogen Burkholderia glumae. Plant Pathol. J. 29:446-450.

Klausner, R. D., Donaldson, J. G. and Lippincott-Schwartz, J. 1992. Brefeldin A: insights into the control of membrane traffic and organelle structure. J. Cell Biol. 116:1071-1080.

Lee, J., Kim, H., Jeon, J.-J., Kim, H.-S., Zeller, K. A., Carter, L. L.A., Leslie, J. F. and Lee, Y.-W. 2012. Population structure of and mycotoxin production by Fusarium graminearum from maize in South Korea. Appl. Environ. Microbiol. 78:2161-2167.

Leslie, J. F. and Summerell, B. 2006. The Fusarium laboratory manual. Blackwell Publishing, Ames, IA, USA.

Punja, Z. K. 1997. Fungal pathogens of American ginseng (Panax quinquefolium) in British Columbia, Canada. Can. J. Plant Pathol. 19:301-306.

Reeleder, R. D. and Brammall, R. A. 1994. Pathogenicity of $P y$ - thium species, Cylindrocarpon destructans, and Rhizoctonia solani to ginseng seedlings in Ontario. Can. J. Plant Pathol. 16:311-316.

Schulte, T. W., Akinaga, S., Soga, S., Sullivan, W., Stensgard, B., Toft, D. and Neckers, L. M. 1998. Antibiotic radicicol binds to the N-terminal domain of Hsp90 and shares important biologic activities with geldanamycin. Cell Stress Chaperon 3:100-108.

Shin, J. H., Yun, B. D., Kim, H. J., Kim, S. J. and Chung, D. Y. 2012. Soil environment and soil-borne plant pathogen causing root rot disease of ginseng. Kor. J. Soil Sci. 45:370-376.

Sweetingham, M. 1983. Studies on the nature and pathogenicity of soil-borne Cylindrocarpon spp. Ph.D. thesis. University of Tasmania.

White, T. J., Bruns, T., Lee, S. and Taylor, J. 1990. Amplification and direct sequencing of fungal ribosomal RNA genes for phylogenetics. Academic Press Inc. New York, USA. 